\title{
reCHERches
}

Culture et histoire dans l'espace roman

$21 \mid 2018$

Studi per Vincenzo Consolo

\section{Conversazione con Vincenzo Consolo}

\section{Anna Frabetti}

\section{OpenEdition}

\section{Journals}

Edizione digitale

URL: https://journals.openedition.org/cher/1155

DOI: 10.4000/cher.1155

ISSN: 2803-5992

\section{Editore}

Presses universitaires de Strasbourg

\section{Edizione cartacea}

Data di pubblicazione: 6 novembre 2018

Paginazione: 11-22

ISBN: 979-10-344-0027-0

ISSN: 1968-035X

\section{Notizia bibliografica digitale}

Anna Frabetti, «Conversazione con Vincenzo Consolo», reCHERches [Online], 21 | 2018, online dal 05 octobre 2021, consultato il 19 novembre 2021. URL: http://journals.openedition.org/cher/1155 ; DOI: https://doi.org/10.4000/cher.1155 


\title{
Conversazione con Vincenzo Consolo
}

\author{
Anna Frabetti ${ }^{1}$
}

Il testo dell'intervista che pubblichiamo appartiene ad un tempo quasi remoto, a una fase della carriera letteraria di Vincenzo Consolo, allora appena sessantenne, in cui la fama ottenuta con Il sorriso dell'ignoto marinaio e con i successivi romanzi lo consacra come uno dei maggiori scrittori italiani della fine del secolo. Il 25 giugno 1994 andai per la prima volta a Milano a incontrarlo e registrai più di due ore di conversazione.

Trascritto minuziosamente dalla diligente studentessa di allora, il testo è rifiutato una prima volta alla pubblicazione o, per meglio dire, è accettato con tanti e tali tagli da indurmi a una giustificata rinuncia. Si perde poi nei meandri di una valanga informatica che travolge il supporto su cui era stato registrato. Per un'incredibile coincidenza, sarà poi ritrovato, su fogli di carta ingiallita, usciti da una preistorica stampante a nove aghi, nel fondo di un cassetto da cui nessuno avrebbe mai potuto stanarlo se non la curiosa allegria di un bambino. In breve, questa la ragione dell'ingiustificabile ritardo di stampa.

Nella revisione del testo ho privilegiato una struttura senza domande, che riordina con la dovuta fedeltà il lungo discorso di Vincenzo Consolo, le argomentazioni e i riferimenti. La continuità del discorso prevale sulla discontinuità della conversazione e permette, a mio parere, di cogliere più estesamente le idee enunciate, come se si trattasse più di una lezione che di un dialogo. Come si vedrà, molti dei temi qui in nuce sono poi stati ripresi e sviluppati altrove, sia nelle numerose interviste, sia negli articoli sia nei saggi ${ }^{2}$. Il discorso si sviluppa dunque intorno ad alcuni blocchi tematici, fitti di rimandi interni, che danno al testo coesione e coerenza. Particolare attenzione è data al nucleo della sperimentazione, all'idea manzoniana della scrittura come «metafora», al rapporto con Pirandello e con Verga.

1 Anna Frabetti, Université de Strasbourg.

2 Si rimanda, a questo proposito, all'importante lavoro che Gianni Turchetta ha svolto per il «Meridiano» dedicato a Consolo: cfr. Consolo 2015. 
Mi auguro, nel chiudere queste pagine, di avere dato la giusta luce alle parole dell'autore, sciogliendo, almeno in parte, un debito di riconoscenza che a lui mi lega, proprio da quel lontano 1994.

\section{II rapporto con le avanguardie, con diverse forme di sperimentazione letteraria}

Antonio Pizzuto non era un autore d'avanguardia, o almeno non militava nell'ultima avanguardia italiana, che - sappiamo tutti - è il Gruppo 63. Ma non è per caso che Pizzuto sperimentava per conto suo ed era arrivato a determinati esiti ed era stato scelto, eletto, come nume tutelare degli avanguardisti del Gruppo 63. E dunque io, mentre cominciavo a scrivere, prendevo le distanze, sia dagli avanguardisti del Gruppo 63, sia da Pizzuto (Cherchi 1987), perché la mia sperimentazione è su un altro crinale, in un altro alveo, ed è la sperimentazione di tipo storicistico di tutti gli scrittori - senza andare lontano nel tempo, parlando del romanzo moderno - a partire da Verga sino a Gadda, passando attraverso Pasolini.

E che cosa significa questo? Significa che lo sperimentatore è colui che lavora sul codice linguistico dato e cerca di rompere questo codice linguistico che, come tutti i codici, diventa rigido nei confronti di quella che Pirandello chiama la Vita. Il compito dello scrittore è di rompere il codice per immettere la Vita, poiché il codice è una Forma. Bisogna rompere tutte le forme per immettere la realtà.

La realtà di Verga, qual era? La realtà di Verga non era mai stata italiana, era una realtà di una periferia geografica, qual era la Sicilia, e di una periferia umana, qual era il grado più basso della condizione umana, quella dei pescatori di Aci Trezza. Ora, per Verga, far parlare questi personaggi in lingua toscana sarebbe stata una contraddizione. Questa è stata la grande rivoluzione linguistica, la grande sperimentazione di Verga. Bisognerebbe fare tutta la storia di Verga, di com'è arrivato a questa sua conversione, a questa caduta da cavallo sulla via di Damasco. Verga è un caso classico di conversione letteraria. Partì imboccando una strada, che è la strada - come sappiamo - del romanzo mondano; esce dalla Sicilia, va a Firenze prima, scrive dei romanzi di successo anche molto conosciuti, ma il romanzo che gli servì da biglietto da visita è stato La storia di una capinera, che era un tema molto alla moda, di derivazione diderotiana e manzoniana, cioè quello della malmonacata. Quindi, quando da Firenze si sposta a Milano, si presenta con questo biglietto da visita molto mondano, viene accolto nei salotti, e pensa di continuare a scrivere i romanzi che aveva letto fino a quel punto, ma si trova in una città che è in un momento storico particolare. Ricordiamoci che era il 1872. Milano era in preda alla prima rivoluzione industriale, la città era in pieno subbuglio, nascevano nuovi quartieri, quartieri operai, nuove industrie, si apriva la Pirelli. Il fatto è che, di fronte a questo sommovimento del panorama sociale, del panorama politico - perché avvenivano allora, con la crescita del capitale, i primi scontri sociali, c'erano i primi scioperi, le prime organizzazioni 
operaie - Verga rimase spiazzato, perché capì che la letteratura che aveva praticato sino a quel momento non rappresentava il contesto storico in cui lui si muoveva. E quindi, come scrive Sapegno, ritornò alla Sicilia della sua infanzia, al mondo intatto e fermo della sua infanzia, che era una sorta di Sicilia immota, la Sicilia del mito, la Sicilia del ricordo. E incominciò qui la sua svolta, la sua grande sperimentazione linguistica, avendo come parametro naturalmente la lingua di Manzoni. Ecco io credo, come credono in tanti, che lui abbia scritto contro Manzoni, contro il toscano di Manzoni.

Ricordiamoci che il toscano di Manzoni era un toscano rivoluzionario, nel momento in cui Manzoni lo scriveva, perché doveva dare una lingua agli italiani; quello di Manzoni era un intento di tipo ideologico, di tipo politico, di unificare questo paese linguisticamente. Quando passa il momento manzoniano, in cui l'unità è avvenuta, in cui la nazione rischiava di diventare nazionalismo, si lasciano fuori dall'indagine letteraria strati popolari e zone di questo paese che la letteratura non aveva riflettuto fino a quel momento, se non a livello dialettale. Verga si incarica quindi di portare in letteratura questi personaggi, che sono i vinti, quelli che lui, nella sua concezione immobile del fato, aveva immaginato come vinti. Parte dal primo gradino della società: concepisce un grande affresco e la rivoluzione linguistica. Da Verga in poi, comincia il filone moderno della letteratura italiana e della sperimentazione linguistica, ma nell'alveo della tradizione.

Quando c'è l'avvento del fascismo - e questo bisogna tenerlo presente perché la letteratura non si muove nel vuoto, si muove nella storia - avviene una sorta di imposizione del codice linguistico nazionale, per cui si scriveva in toscano e il romanzo di tipo sperimentale incominciò a diventare sospetto. Si formarono delle correnti letterarie che vanno sotto il nome di Ronda o di Voce, che immaginavano una sorta di lingua pura, di lingua metallica, assolutamente formale. Il romanzo cadde in disuso e venne di moda il frammento, ci fu il periodo del frammentismo, dell'elzeviro, che era la bella prosa.

Non bisogna poi dimenticare le due esperienze che precedono il fascismo e sono parallele ma speculari: da una parte, questa specie di orgia linguistica e lussureggiante che era stata la lingua dannunziana e che aveva pervaso tutta la borghesia e la piccola borghesia italiana, per cui, quando si scriveva, bisognava scrivere secondo i moduli dannunziani. Dall'altra parte, c'era stata l'esperienza devastante, azzerante dei futuristi, dei marinettiani. Si tratta di due fenomeni speculari: voglio dire che tutti e due, sia Marinetti che D'Annunzio, non facevano altro che riflettere la lingua del potere e al potere interessava azzerare $\mathrm{i}$ codici della lingua per poter costruire una lingua del potere stesso.

Io penso veramente che le avanguardie non siano altro che la faccia speculare del codice linguistico del potere; quando poi finiscono, decadono, adottano la lingua del potere, la lingua della conformazione. Mentre la sperimentazione è più inquieta, perché opera nella tradizione e cerca di immettere le voci della verità, non delle voci artificiali, di gruppi di letterati. Finito il fascismo, crollata 
questa doppia lingua, Pasolini fa un'analisi molto dettagliata e molto bella nel 1964, quando scrive il suo famoso saggio sulla nascita della lingua italiana e fa una disamina di quel che era la lingua italiana fino a quel punto ${ }^{3}$.

Caduto il fascismo, naturalmente si sente l'esigenza di tornare alle vecchie sperimentazioni, con immissioni, con sperimentazioni linguistiche, e si ha il grande fenomeno di Gadda. Non si può immaginare niente di più lontano di uno scrittore come Gadda da Verga. Verga è casto, parco, asciutto: la sua lingua non era dialettale, ma era - come dice Pasolini - una lingua toscana irradiata di dialettalità; aveva abbassato il codice linguistico toscano a livello della sintassi e della paratassi dialettale siciliana, mettendo in campo tutti i modi di dire, $\mathrm{i}$ proverbi. Verga aborriva il dialetto, perché sapeva bene che il dialetto era un'altra cosa. Aveva avuto una polemica con Capuana sull'uso del dialetto in letteratura, e non solo con Capuana, ma anche con un altro scrittore siciliano che si chiama Alessio Di Giovanni, che gli aveva proposto di tradurre in siciliano I Malavoglia. Verga si era assolutamente opposto, perché sapeva qual era la sua rivoluzione linguistica. Parlando appunto di Gadda, sono due scrittori molto lontani: quanto uno è monocorde, così parco, così pietroso, tanto l'altro è polifonico, ricco, barocco. Gadda, in un'intervista uscita in un libro recente (questa intervista credo sia uscita al momento della pubblicazione del Pasticciaccio brutto de via Merulana), a chi gli faceva i nomi di Zola, di Queneau, di altri scrittori francesi, di una sperimentazione esterna all'Italia, ha risposto di considerarsi figlio di Verga. Si riconosceva appunto in questo filone della sperimentazione linguistica italiana a partire da Verga, solo che gli strumenti usati da Gadda erano diversi da quelli usati da Verga. In Gadda c'è la polifonicità dei dialetti: recupera tutti i dialetti italiani (questo grande calderone) per rappresentare il paese, mentre Verga usava un registro monocorde dell'irradiazione.

Pasolini, insieme a Gadda, è stato l'autore che nei romanzi ha sperimentato anche linguisticamente attraverso la tecnica della digressione; i personaggi partivano dall'italiano, poi man mano scivolavano verso il dialetto. Io mi riconosco in questo filone, considero come mio padre tutelare Verga, non credo negli azzeramenti, non credo nelle avanguardie, perché credo che la letteratura sia una continua sperimentazione, sperimentazione non solo linguistica, ma anche sperimentazione sulle strutture narrative, sulla struttura stessa del romanzo. Per quanto mi riguarda, io non ho usato né la digressione pasoliniana o gaddiana né l'irradiazione di tipo verghiano. Io ho usato quello che chiamerei l'innesto: innesto di parole, di fonemi, di lessici, che sono stati espunti dal codice linguistico nazionale, che mi ritrovo nella memoria, nel mio bagaglio regionale, nella storia linguistica siciliana. Parole di lingue che le varie dominazioni hanno lasciato in Sicilia, le varie civiltà se non vogliamo chiamarle dominazioni. E quindi io cerco di immettere questi vocaboli, che non sono italiani ma che hanno una loro dignità filologica, che vengono da altre lingue. Sono vocaboli che di volta in volta possono venire dal latino o dal greco, dall'arabo o dal francese,

3 Si tratta naturalmente di Nuove questioni linguistiche; cfr. Pasolini 1964. 
dallo spagnolo e che sono il segno della ricchezza storica, civile e linguistica della regione di cui io parlo. I miei personaggi, e anche lo stesso io narrante nei miei romanzi, recuperano questi vocaboli che sono stati cacciati via, che sono stati sepolti.

Io ho spesso paragonato il mio lavoro di ricerca linguistica al lavoro dell'archeologo: cerco di scavare per tentare di disseppellire questi resti, questi reperti linguistici, e di metterli alla luce, di metterli in circolo. Poi, naturalmente, obbedisco ad altri criteri: sono i criteri della sonorità, del polisenso che può avere un vocabolo di più sensi piuttosto che un unico senso. Ubbidisco a tante esigenze in questa mia sperimentazione. In questo mi sento lontano da Pizzuto, perché io sono sempre spinto da un intento storicistico nella mia ricerca, cerco di essere sempre aggrappato alla storia e la mia sperimentazione non è solamente in senso formale. Mi preoccupo sempre che ci sia un equilibrio tra il contenuto e la forma, tra quello che voglio dire e il modo in cui lo dico. In Pizzuto invece c'era lo scivolamento solo in un senso formale, e la sua scrittura diventava come una musica atonale, una scrittura astratta, dove la linea narrativa non si svolgeva più, le parole diventavano puro suono senza il significato, era puro significante. In quello mi sento molto lontano da Pizzuto e capisco perché gli avanguardisti avessero scelto Pizzuto come loro nume tutelare, proprio perché era la pura sperimentazione formale.

\section{II rapporto con Luigi Pirandello}

La lezione pirandelliana l'ho appresa attraverso Sciascia. Credo che il mio nome si possa accostare a Pirandello in questo: che c'è in me l'ansia... Voglio dire che c'è in me il desiderio, il bisogno, la volontà di uscire dall'immobilismo verghiano; perché io credo che in Sicilia ci siano due modi per essere scrittori: appartenere al filone verghiano o appartenere al filone pirandelliano. Voglio dire che ci si può immettere nella zona del mito, ci si può immettere nella zona dei temi dell'assoluto dell'esistenza e chiudersi in questa sorta di concezione fatalistica della vita, dell'esistenza della condanna del fato nel modo in cui l'aveva concepito Verga, di una vita circolare senza assolutamente nessuna via di uscita. Io credo che la modernità di Pirandello consista nell'aver cercato di rompere questo cerchio e di riportare il discorso letterario non sulla circolarità, ma sulla linearità. E Pirandello l'ha fatto attraverso la dialettica, l'ha fatto attraverso la parola, quindi ha rotto quella chiusura del proverbio, del modo di dire ereditato dai padri, dalla tradizione, così come l'aveva concepito Verga, questo parlare come se fosse un salmodiare di Sacre Scritture, della Bibbia o del Corano; erano delle formule quasi sacre che si ripetevano. Pirandello ha cercato di far uscire l'uomo dalla sua condizione di condanna attraverso la ribellione, la rottura di questo codice linguistico e quindi la dialettica, l'interrogarsi sul perché di questa condanna, il chiedere conto a qualcuno del perché di questa condanna. $\mathrm{Ne}$ viene fuori un mondo ancora più straziante in Pirandello: è quello che Giovanni Macchia chiama «la stanza della tortura» (Macchia 1981), è un continuo 
torturarsi. C'è questa grande rivoluzione pirandelliana nel teatro e nella sua prosa - a parte i romanzi di tipo storico - di uscire dal cerchio verghiano e di rompere la fatalità della tragedia greca, di portarla sul piano della commedia moderna attraverso l'umorismo, attraverso l'ironia, attraverso l'articolazione della parola e del discorso, quindi attraverso la comunicazione. Ora, questo è un modo illuministico, un modo moderno di concepire la condizione dell'uomo e io ho cercato, per il mio destino di centralità - sono nato nella Sicilia centrale e mi sono trovato in bilico tra questi due mondi, il mondo orientale di Verga, che è invaso dalla natura, che protende verso il lirismo e verso il mito, e il mondo occidentale, che è il mondo più dialettico, il mondo più storicistico, più della ragione, a cui appartiene Pirandello, a cui appartiene Sciascia - io ho cercato di conciliare questi due opposti e quindi la mia scrittura forse consiste in questa continua oscillazione tra il mito e la storia, tra il lirismo e la dialettica. Io, sotto questo bisogno dell'espressività o dell'espressionismo, come l'ha chiamato Segre, credo che si scorga il martellare della ragione, della dialettica, dello storicismo. In questo io mi sento anche erede di Pirandello. Per esempio Vittorini, che apparteneva alla Sicilia orientale - veniva da Siracusa - e quindi era fortemente invaso anche lui dalla natura, aveva una propensione al lirismo, che non poteva spegnere; anche lui aveva sentito il bisogno di uscire dall'immobilità, aveva concepito i suoi racconti, i suoi romanzi come viaggi, come movimento; però l'aveva fatto soltanto esteriormente, perché poi anche Vittorini, alla fine, risulta un grande formalista. Tuttavia c'era questa esigenza del movimento, del fare, del togliersi dall'immobilità della rassegnazione, del dolore. Mentre in Pirandello tutto questo dolore diventa essenza, diventa parola, in Vittorini è soltanto nella vicenda raccontata il movimento, ma formalmente, stilisticamente, rimane chiuso anche lui dentro la forma.

\section{II teatro, la teatralità, la «vastasata»}

Pirandello per forza doveva poi alla fine salire sul palcoscenico, perché la sua scrittura è teatrale, è una scrittura dialettica, dialogica, fortemente teatrale; quindi ha sentito il bisogno del teatro e di rompere le pareti del teatro, di creare la quarta dimensione.

La «vastasata» era il teatro popolare, molto scurrile, per i facchini, perché «vastaso» viene da «bastizo», che in greco significa «portare addosso» e quindi da noi esiste questa parola "vastaso" che è detta in doppio senso: sia come professione («u vastasu» è colui che porta, il facchino), ma anche nel senso metaforico della parola, che vuol dire sporcaccione, colui che usa il linguaggio scurrile. C'era quindi una forma di teatro siciliano, soprattutto palermitano, che si chiamava la "vastasata», che era un po' come le atellanae, come il teatro romano più scurrile. Cocchiara (1926) si è occupato di questa forma di teatro, che usa un linguaggio molto sboccato, molto diretto, un teatro di tipo carnascialesco, che poi usciva dagli argini del carnevale e diventava un teatro per tutto l'anno, 
perché quella licenziosità si ammetteva soltanto nel periodo del carnevale, ma poi ha finito per essere recitato tutto l'anno.

\section{Pirandello, Sciascia e lo zolfo}

Essere dentro o fuori dalla miniera di zolfo significa che i parenti di Pirandello erano proprietari di zolfare e Sciascia era nipote di uno zolfataro. La differenza sta in questo. Pirandello stesso ha fatto il guardiano per un breve periodo, quando voleva smettere di studiare, in una zolfara. Suo padre era un proprietario e commerciante di zolfo e forse il nucleo del dolore pirandelliano viene da questa personalità del padre, che era molto forte, e da questo mondo tremendo e anche terribile dei commercianti di zolfo, dei proprietari. Sono tutti e due scrittori di tipo logico-dialettico, ma l'uno verso la zona dell'esistenza (Pirandello), l'altro verso la zona civile, politica, perché anche tutta la letteratura di Sciascia è una letteratura dialettica, quella dell'indagine, del delitto ecc.

È questa la grandezza di Pirandello. Dove avviene questa tortura? Nel chiuso di una stanza, in un interno borghese, mentre la dialettica di Sciascia avviene nella piazza del paese di Racalmuto, dove si discute dell'amministrazione comunale, del circolo dei civili, perché l'uno era figlio di zolfataro, l'altro era figlio di proprietari dello zolfo. È questa la differenza per cui io dico «dentro» o «fuori» della miniera. Erano tutti e due sulfurei, sia Pirandello che Sciascia. La presenza dello zolfo è stata molto importante in questi due scrittori, credo che abbia veramente segnato le loro personalità. Io ho scritto un saggio sulla letteratura dello zolfo, uscito in un libro che si chiama ' $N$ fernu vero, delle Edizioni del Lavoro (Grimaldi 1985). È una di quelle cose che dovrò ripubblicare. È una disamina di quella che è stata la letteratura scaturita nella zona delle zolfare in Sicilia.

\section{La parodia, I'«abrasione»}

Il mio romanzo su cui i critici si sono più soffermati, Il sorriso dell'ignoto marinaio, nella scrittura è la parodia di una scrittura, non è la scrittura dell'autore: è la parodia di un erudito dell'Ottocento, che è il barone di Mandralisca, che poi cade in crisi. È, dicevo io, una scrittura in negativo perché, nel momento in cui questa scrittura si dichiara, nello stesso tempo si nega, perché è parodistica, perché è abrasiva, l'intento è abrasivo. E quindi, a un certo punto, c'è la rottura di questo linguaggio ottocentesco, dell'erudizione ottocentesca, con le scritte dei condannati a morte, c'è questo stacco linguistico, questa frattura. Il libro è molto complesso, perché bisognerebbe parlare anche della struttura oltre che del linguaggio. Questi giochi sono però sempre in bilico, perché è una scrittura in negativo che a momenti, nei momenti di commozione, si rovescia in scrittura in positivo, nella rievocazione degli orrori; quando il barone descrive la strage di cui è stato testimone, c'è molta pietà e allora la parodia si smette, diventa adesione, non è più parodia. 
Anche Retablo è il massimo della parodia, è una parodia settecentesca di Fabrizio Clerici, che è un uomo del nostro tempo. Ma sotto questa parodia vengono fuori dei momenti di grande sarcasmo nei confronti di una certa Sicilia retorica, che è quella di Alcamo. Poi c'è naturalmente la commozione di fronte a una certa bellezza della Sicilia. Questo è un libro che nasce dalla polemica: è l'allontanamento di un intellettuale, di un artista, da Milano, da una Milano fortemente ideologica che in quel momento - era la Milano degli Illuministi - ed era la polemica di chi scriveva, contro il cristallizzarsi solamente dell'ideologia, che riflette solo il dato politico dell'uomo e non il momento globale umano. E questo personaggio, innamorato di Teresa Blasco (dentro ci sono molti segni, molte metafore), che poi è la nonna di Manzoni, il padre della letteratura italiana moderna, ha questo senso: che l'ideologia con la poesia (che è rappresentata da Teresa Blasco) genera uno scrittore come Manzoni. E quindi Fabrizio Clerici che si allontana dalla città ideologica, dalla città illuministica, va alla ricerca della poesia e la cerca nel luogo in cui è nata la sua donna e quindi prova commozione di fronte alla bellezza, ma vede anche gli orrori, vede quello che deve vedere la letteratura, che nessuna ideologia, nessuna storiografia riesce a vedere. Soltanto l'arte riesce a vedere gli orrori e le bellezze dell'esistenza, della vita, quindi ecco le scene di Alcamo, la commozione di fronte alle antichità, che a volte sono anche ironizzate; ad esempio, quando c'è la statua dell'efebo di Mozia che cade in mare, c'è l'ironia del feticismo delle antichità, ci sono tutti questi segni. È anche quello un libro molto parodistico, che vuole liberare determinate metafore moderne; poi c'è un'invettiva contro Milano da parte di Fabrizio Clerici, che era un'invettiva di uno - di chi scrive - che aveva amato questa città, che se ne era disamorato perché vedeva questa città deteriorarsi, diventare quello che sappiamo. C'è un momento in cui lui dice "arrasso, arrasso», in cui c'è l'enumerazione di una Milano che era la Milano del momento in cui io scrivevo e in cui tutti i personaggi che lui cita nella sua invettiva sono riconoscibili; quando parla del politico ladro, del prete trafficone, del poeta decadente, del gazzettiere: c’è tutta la Milano degli anni Ottanta, la Milano di Craxi.

La dialettica Milano-Sicilia era molto più ridotta prima, era la dialettica - fin dal mio primo libro - tra la Sicilia orientale e la Sicilia occidentale. Io sono sempre stato dilaniato tra due polarità. Poco fa, facevo l'esempio di Verga e Pirandello; questo bisogno di conciliare gli opposti l'ho sempre sentito, allontanandomi dalla Sicilia. Retablo è proprio molto emblematico in questo senso, di questa polarità più ampia Milano-Sicilia. Dovrebbe essere il mondo della politica, della ragione, del vivere civile con il mondo del mito, della poesia, del lirismo, con tutto quel che significa, in un certo senso anche la Sicilia, il mondo verghiano; perché Pirandello aveva preso, anche se stava ad Agrigento, le distanze dalla Sicilia, per poterne parlare in modo logico, in modo razionale, dialettico. 


\section{La scrittura, la distanza memoriale}

In Retablo c'è anche l'idea dello scrittore come «castrato» della vita. È la ripresa della frase di Pirandello «la vita o la si scrive o la si vive». È l'idea della condizione dell'artista, di questa nostalgia struggente di una vita da cui ci si apparta continuamente, e quindi c'è il tendere la mano, sempre per cercare di afferrare la vita che, intanto, mentre tu ti apparti per tentare di rappresentarla, scorre per conto proprio.

L'idea su cui si fonda Retablo è quella della lontananza che consente la scrittura, l'idea della distanza memoriale. Poi lo scrittore si riattualizza compiendo quel salto mortale che è la metafora, perché l'artista - lo scrittore soprattutto - ha sempre bisogno di volgere la testa indietro per attingere alla memoria, a quel patrimonio che si porta dietro e che non è l'attualità ${ }^{4}$ Questa necessità di volgere la testa indietro per attingere alla memoria è la distanza memoriale. Io non concepisco letteratura senza memoria. Non capisco tutta la querelle stupida che c'è stata negli anni scorsi tra i giornalisti, che sono i nuovi scrittori perché parlano dell'attualità, e degli scrittori che sono inattuali. C'è un grande discorso da fare su questo momento che stiamo vivendo, su questo secolo in cui c'è stata l'esplosione dei mezzi di comunicazione di massa e quindi la messa in crisi dello scrittore. E lo scrittore, per difendere lo spazio letterario, deve sempre di più ricorrere alla memoria, perché non è possibile per uno scrittore raccontare in termini assolutamente di comunicazione l'attualità, raccontarla in modo così diretto come fanno i giornali ogni mattina o come fa la televisione. Perciò molto spesso si confonde il giornalismo con la letteratura e molti aspirano a far omologare, a far diventare la letteratura giornalismo.

Quando è uscito il mio ultimo libro, Nottetempo, casa per casa, c'è stato un famoso giornalista, il quale ha una rubrica su L'Espresso, che ha osservato con molto candore:

Ma questo Consolo, quando scrive degli articoli sui giornali è chiarissimo, molto logico, e quando scrive i romanzi usa dei vocaboli come questi...

e indicava tutta una sfilza di vocaboli che a lui sembravano strani, molto ricercati. Si chiedeva il perché di questa dicotomia, di questa disparità, e metteva il dito come si dice - nella piaga, cioè non capiva la differenza tra quella scrittura che Barthes chiama «scrittura d'intervento», la scrittura giornalistica, e la scrittura letteraria. Non vedeva la necessità dell'altra scrittura perché lui, da giornalista, vorrebbe che la letteratura fosse come il giornalismo.

4 Consolo 1993: 46-47: «E noi scrittori siciliani, "inclini” alla storia, troviamo in Manzoni paternità e sostegno. Nel Manzoni dei Promessi sposi e della Colonna infame, quello della necessità della storia, prima della narrazione e soprattutto quello della necessità della metafora. [...] La lezione di Manzoni è proprio la metafora. Ci siamo sempre chiesti perché abbia ambientato il suo romanzo nel Seicento e non nell'Ottocento. Oltre che per il rovello per la giustizia, proprio per dare distanza alla sua inarrestabile metafora. L'Italia del Manzoni sembra davvero eterna, inestinguibile». 
Tutto ormai deve diventare giornalismo e anche questi grandi giornalisti, come Bocca o come Pansa, dicevano qualche anno fa: "Siamo noi i nuovi narratori, i nuovi Balzac». Il loro è un linguaggio passivo, d'accatto, sono dei gerghi che poi si scimmiottano, si ripetono. Ci sono delle formulette che inconsciamente, naturalmente si trasmettono l'uno con l'altro e ripetono passivamente perché loro non hanno un problema di linguaggio, quindi hanno bisogno di usare un linguaggio che è già consumato, per essere il più comunicativi possibile. E stiamo parlando di parole, non consideriamo quello che è il mondo delle immagini...

\section{Il rapporto fra segni verbali e segni iconici}

Nel Sorriso dell'ignoto marinaio la figuratività è portata proprio come una cifra, c'è l'iconografia dell'ignoto marinaio. In Retablo la topologia figurativa è fondata poeticamente sull'assenza di un referente concreto. In tutto il libro c'è un'interruzione continua quando Isidoro deve parlare di Rosalia, c'è sempre un'interruzione, perché poi c'è il disvelamento alla fine. Rosalia rimane il vagheggiamento, l'eterno femminino, rimane anche l'ambiguità dell'altro, perché la parola veritas è una parola naturalmente ironica, perché la verità è quella effigiata dallo scultore Serpotta, la verità nuda, prorompente; ma questa fanciulla, che è molto ambigua - non si capisce se abbia amato Isidoro o se l'abbia ingannato, preso in giro, giocato -, è poi l'inganno dell'amore, l'inganno della poesia, che però sono degli inganni assolutamente necessari all'uomo, senza i quali non potremmo vivere. La citazione iniziale di Jacopo da Lentini evidenzia già tutto il tracciato del libro. L'uso di una certa topologia figurativa, del riferimento a una tradizione «alta» della poesia, è continuamente ribaltato, rovesciato, a fini parodici.

In Retablo, già la parola «retablo» è iconica, è una composizione pittorica e nello stesso tempo è anche spettacolo teatrale. In Cervantes, il retablo diventa lo spettacolo teatrale; infatti c'è l'illusione del «retablo de las maravillas», il nome è preso da Cervantes. Nella tradizione pittorica siciliana che ci hanno portato gli spagnoli, c'è la parola «retablo», una composizione pittorica in più riquadri, dove si racconta una storia. Ma c'è anche questo teatrino delle illusioni che è la letteratura. E quindi Rosalia è l'interprete principale di questo teatrino. Il primo capitolo e l'ultimo, che sono i pannelli laterali del retablo, seguono lo schema del contrasto, del contrasto d'amore, con le due voci - prima Isidoro, poi lei, Rosalia, che risponde, come in Rosa fresca aulentissima.

Altro dato importante è il colorismo. La pesca del corallo è una cosa molto mediterranea, soprattutto Trapani era uno dei centri della pesca del corallo, dove c'era un grande artigianato e quindi c'è l'amore di tutta questa memoria, ma anche di questa materia marina; è una concrezione marina il corallo, che ricorda molto la grazia femminile, con questo rosa acceso che dà luminosità al volto delle donne, più degli smeraldi che sono molto freddi, freddi come le stelle; è una pietra più carnale. 
Il corallo che viene usato per la descrizione di Rosalia, come il verde che viene usato per Teresa Blasco, alla fine si fondono. Rosalia amata da Isidoro, Rosalia amata da Vito Sammataro, Teresa Blasco, alla fine diventano un'unica immagine, un solo profilo. C'è un momento in cui Fabrizio Clerici disegna il profilo di una donna e ognuno vede la propria Rosalia: è l'idea propria di tanta poesia italiana, almeno fino al Cinquecento, e di tanta lirica a sfondo teologico, l'idea della rappresentazione dell'assenza, dell'irrealizzazione.

\section{La religione, la religiosità}

Nei miei libri c'è una sorta di critica alla religione come potere, anche alla condizione clericale come condizione innaturale, perché i miei preti e i miei frati molto spesso impazziscono, perdono i freni. Anche nelle Pietre di Pantàlica c'è un altro frate pazzo (oltre a frate Nunzio, frate Isidoro, fra' Giacinto), Frate Agrippino, che si fustiga. C’è questa innaturalità della costrizione del potere della religione sull'uomo, però c'è anche il recupero di una religiosità più autentica, più armonica con la vita, con il mondo, che è quella del pastore di Segesta; quello è un luogo pregreco, dove poi sono arrivati i Greci, dove c'erano gli Elimi e quindi c'erano dei templi di dee catactonie, sotterranee, dove si facevano dei sacrifici con gli elementi naturali, con il latte, con il miele. Poi c'è stata una sorta di sincretismo con l'arrivo dei Greci, del recupero di queste forme epocali, arcaiche. E quindi c'era il bisogno di una religione che fosse più consona con la natura, meno di frattura con la natura, di una religione che non fosse un apparato di potere, che non fosse costrizione. C'è questo, non il gusto di essere dissacratore. Il racconto dei frati nel convento della Gancia è una sorta di parodia boccaccesca, un po' grottesca, un po' atroce. Anche don Gregorio Nanfara, in Filosofiana, è un esempio di impostura. Don Gregorio era l'impostore locale, che era stato in seminario, sapeva un po' di latino e imbrogliava la povera gente come Vito Parlagreco, perché quest'ultimo era uno che cercava il tesoro dentro questa tomba, mentre il furbo don Gregorio sapeva che il tesoro erano i vasi, di cui si appropria e che poi avrebbe rivenduto. È sempre l'idea del più acculturato che frega il più ignorante, con tutte le sue formule latine, con i suoi libri, vivendo di questa mistificazione, di questa impostura; quindi è la denuncia della religione come impostura, come acquisizione di determinate formule per ingannare.

Nel Sorriso dell'ignoto marinaio c'è poi l'idea manzoniana della scrittura come potere, come impostura. La scrittura come strumento del potere, nel Sorriso è questo. Manzoni fa dire a un popolano la stessa cosa; infatti Renzo è stato fregato dal latino di don Abbondio e da Azzeccagarbugli.

\section{Bibliografia}

Cherchi G., 1987, «Mille e una notte», L'Unità, 11 novembre.

Cocchiara G., 1926, Le vastasate, Palermo, Sandron. 
Consolo V., 1993, Fuga dall'Etna. La Sicilia e Milano, la memoria e la storia, Roma, Donzelli.

Consolo V., 2015, L'opera completa, a cura e con un saggio introduttivo di Gianni Turchetta e uno scritto di Cesare Segre, Milano, Mondadori, «I Meridiani». Grimaldi A., 1985, Nfernu veru. Uomini e immagini dei paesi dello zolfo, saggio introduttivo di Vincenzo Consolo, Roma, Edizioni del Lavoro.

Macchia G., 1981, Pirandello o la stanza della tortura, Milano, Mondadori.

Pasolini P.P., 1964, «Nuove questioni linguistiche», Rinascita, 26 dicembre. 